\title{
Efeito de Dois Regimes de Suplementação Alimentar e Dois Sistemas de Produção, nos Constituintes Sangüíneos de Cabras Saanen Durante a Lactação ${ }^{1}$
}

\author{
Adriana Sampaio Nunes ${ }^{2}$, Orlando Rus Barbosa ${ }^{3}$, Eduardo Shiguero Sakaguti ${ }^{3}$, Marlene Leiko \\ Doi Sakuno ${ }^{4}$, Maria de Fátima T. E. de Araujo ${ }^{5}$, Cilene Pereira da Silva ${ }^{6}$
}

RESUMO - Foram analisados os constituintes sangüíneos de oito cabras leiteiras cruza Saanen lactantes, entre novembro (1997) e maio (1998), submetidas a dois sistemas de produção - pastagem (S1) e semiconfinamento (S2) - e dois regimes alimentares: $30 \%$ das exigências em energia líquida ao longo de toda a lactação (R1) e $60 \%$ das exigências diárias em energia líquida da $3^{\mathrm{a}}$ a $13^{\mathrm{a}}$ semana de lactação e $15 \%$ das exigências em energia líquida da $14^{\mathrm{a}}$ semana até o final da lactação (R2). Amostras de sangue foram coletadas quinzenalmente de dois animais de cada tratamento para a análise de glicose (Gl), hemoglobina (Hb) e hematócrito (Ht). Não foram encontradas diferenças na concentração de GL e Hb e no hematócrito, nos diferentes regimes alimentares. Os animais do sistema de produção $\mathrm{S} 1$ apresentaram maiores valores de $\mathrm{Hb}(7,60 \mathrm{~g} / 100 \mathrm{~mL})$ e $\mathrm{Ht}(22,42 \%)$ em relação aos animais do sistema de produção $\mathrm{S} 2(\mathrm{Hb}=6,05 \mathrm{~g} / 100 \mathrm{~mL}$ e Ht $=$ $17,71 \%)$, não havendo diferença entre os dois sistemas de produção em relação à Gl.

Palavras-chave: glicose, hemoglobina, hematócrito, sistema de produção, suplementação alimentar

\section{Effect of Two Regimes of Alimentary Supplementation and Two Systems of Production on Blood Traits of Dairy Goats Bred}

\begin{abstract}
Three blood constituents in eight crossbred Saanen goats bred in two systems - pasture (S1) and semi-confinement (S2) - were analyzed. The animals were fed two different diets: $30 \%$ of the dairy requirements of net energy supplied by concentrate during all the lactation (R1), and $60 \%$ of the requirements from $3^{\text {rd }}$ to $13^{\text {th }}$ week of lactation and $15 \%$ from $14^{\text {th }}$ until the end of lactation (R2). Blood samples were taken to evaluate glucose (Gl), hemoglobin (Hb), and hematocrit (Ht) levels. The results showed that none of these traits were affected by the feeding systems. Animals in the S1 production system presented higher values of $\mathrm{Hb}(7,60 \mathrm{~g} / 100$ $\mathrm{mL})$ and $\mathrm{Ht}(22,42 \%)$ than those of S2 animals $(\mathrm{Hb}=6,05 \mathrm{~g} / 100 \mathrm{~mL}$ and $\mathrm{Ht}=17,71 \%)$. No significant difference was observed between the two production systems for Gl.
\end{abstract}

Key Words: climatic variables, glucose, hemoglobin, hematocrit, production systems, and supplementation diet

\section{Introdução}

O organismo dos animais homeotérmicos pode ser considerado uma máquina térmica, sendo sua energia conseguida a partir de fenômenos térmicos. A energia produzida pelo organismo animal advém de reações químicas internas, sendo a mais importante a combinação do carbono, produzida no organismo sob forma de alimentos, com o oxigênio extraído do ar pela respiração. Esse processo de produção de energia interna, a partir de elementos combustíveis orgânicos, é denominado metabolismo. O organismo adquire energia pelo metabolismo. Tanto o calor produzido como o dissipado depende da atividade desenvolvida pelo animal (Nããs, 1989).
Nos grandes animais com elevada taxa metabólica, o sangue é essencial para o transporte de calor, caso contrário seus órgãos internos rapidamente ficariam superaquecidos (Schmidt-Nielsen, 1996). O sangue assimila nos órgãos em atividade o calor formado durante os processos metabólicos, distribuindo-o por todo o corpo e, devido à sua mediação, processa-se na pele e nos pulmões a liberação de calor. Alterações na temperatura do sangue são estímulos importantes para os centros de regulação térmica situados no hipotálamo (Güntler et al., 1986).

O sangue é o veículo de comunicação entre os órgãos, transportando nutrientes, oxigênio dos pulmões para os tecidos e dióxido de carbono gerado durante o metabolismo respiratório dos tecidos para

\footnotetext{
1 Parte da tese de mestrado do primeiro autor.

2 Estudante do curso de Pós-Graduação em Zootecnia UEM.

3 Professores DZO - UEM - Maringá, PR. E.mail: orbarbosa@uem.br; essakaguti@uem.br

4 Professora DAC - UEM - Maringá, PR.

5 Farmacêutica Setor de Hematologia - UEM Maringá, 6 Técnica Setor de Hematologia - UEM Maringá.
} 
excreção pulmonar. Além disso, os hormônios são transportados pelo sangue das glândulas endócrinas para os órgãos-alvo específicos, na sua função de mensageiros químicos. A composição química sangüínea é complexa, porque através do sangue é transportado grande número de nutrientes metabólicos e, ainda, de produtos residuais e íons orgânicos, tornando possíveis a intercorrelação coordenada e a integração do metabolismo em vários órgãos dos animais superiores (Lehninger, 1984).

A glicose durante a lactação é metabolizada a processos metabólicos da glândula mamária (Reynolds et al., 1994).

Quanto maior a solicitação física de um animal, maior será o valor do hematócrito. A hemoglobina é responsável pelo transporte de oxigênio dos pulmões para os demais tecidos. Sabendo-se que a função da hemoglobina consiste no transporte de oxigênio dos pulmões para os diferentes tecidos, durante o esforço físico a liberação de $\mathrm{O} 2$ é mais rápida, em função da elevação na taxa de consumo de oxigênio (SchmidtNielsen, 1996).

O presente trabalho foi conduzido com o objetivo de avaliar o efeito de dois regimes de suplementação alimentar e dois sistemas de produção sobre os teores de glicose e hemoglobina e o valor do hematócrito no sangue de cabras Saanen, durante o período de lactação.

\section{Material e Métodos}

O experimento foi realizado no setor de Caprinocultura da Fazenda Experimental de Iguatemi (FEI), pertencente à Universidade Estadual de Maringá (UEM), no período de novembro de 1997 a maio de 1998.

Foram analisados os constituintes sangüíneos de duas cabras de cada tratamento, totalizando oito animais com grau de sangue variado $(1 / 2,3 / 4,7 / 8 \mathrm{e}$ 15/16) da raça Saanen, primíparas e multíparas, em lactação. Foi utilizado um abrigo, dividido em boxes individuais contendo bebedouro, cochos e canzis e uma área de pastagem de estrela africana (Cynodon nienfuensis). $\mathrm{O}$ abrigo era constituído por paredes e piso ripado com dimensão de $20 \mathrm{~m} \times 9 \mathrm{~m} \times 2 \mathrm{~m}$, coberto com telha de fibra cimento amianto, com orientação do eixo maior da cobertura no sentido norte-sul.

Os tratamentos testados foram dois tipos de sistemas de produção - em pastagem (S1) e semiconfinamento (S2) - e dois regimes de forneci- mento de concentrado: $30 \%$ das exigências em energia líquida ao longo de toda a lactação (R1) e $60 \%$ das exigências diárias em energia líquida da $3^{\mathrm{a}}$ à $13^{\mathrm{a}}$ semana de lactação e $15 \%$ das exigências em energia líquida da $14^{\mathrm{a}}$ semana até o final da lactação (R2) (NRC, 1981). Os animais pertencentes ao sistema S2 tiveram acesso a um abrigo, dividido em boxes individuais contendo bebedouro, cochos e canzis e a uma área de pastagem de estrela africana (Cynodon nienfuensis). Os animais pertencentes ao sistema S1 tiveram acesso somente à área de pastagem. A quantidade de concentrado fornecida foi corrigida semanalmente, de acordo com a produção de leite e o peso vivo dos animais. Os concentrados fornecidos aos animais em crescimento e aos adultos continham $72 \%$ de milho e $28 \%$ de farelo de soja, $75 \%$ de milho e $25 \%$ de farelo de soja, respectivamente. Foi considerado animal em crescimento aquele da primeira ou segunda lactação e com peso corporal inferior ao peso adulto esperado $(60 \mathrm{~kg})$. Os animais foram ordenhados pela manhã (6h) e à tarde (15h30). Após a ordenha da manhã, as cabras receberam concentrado, na quantidade determinada, e, logo após, levadas à pastagem. Depois da ordenha da tarde, cada animal recebia a quantidade determinada de concentrado, sendo que os animais do sistema semiconfinamento foram recolhidos ao abrigo, onde permaneceram durante a noite recebendo feno de gramíneas do gênero Cynodon à vontade. Os animais do sistema S1 permaneceram durante todo o período na pastagem, tendo livre acesso ao abrigo durante a noite sem receber qualquer tipo de suplementação. Água e sal mineral foram fornecidos à vontade.

$\mathrm{Na}$ coleta dos dados de velocidade do vento, utilizou-se um termoanemômetro. As temperaturas e a umidade do ar foram obtidas por intermédio de um psicrômetro não ventilado de bulbo seco e bulbo úmido. Para obtenção do calor radiante, foi utilizado um globo de Vernon de 0,15 m de diâmetro (Silva, 1989) exposto ao sol. A coleta de dados das variáveis meteorológicas foi efetuada duas vezes por semana, em intervalos de uma hora, das 8 às $18 \mathrm{~h}$. A partir dos dados de temperatura e umidade do ar, foi calculado o índice de temperatura e umidade (ITU), mediante a fórmula referida por Thom (1958):

$$
\mathrm{ITU}=\mathrm{Ta}+0,36 \mathrm{Tpo}+41,4
$$

em que: $\mathrm{Ta}=$ temperatura do ar, $\left({ }^{\circ} \mathrm{C}\right) ; \mathrm{Tpo}=$ temperatura do ponto de orvalho $\left({ }^{\circ} \mathrm{C}\right)$.

Após a obtenção do ITU, consideraram-se as seguintes variáveis: ITU médio máximo e mínimo, 
levando-se em consideração a interpretação dos dados segundo Hahn (1985), emque número de dias com ITU inferior a 70 representa ausência de estresse; entre 70 e 72, alerta, alcançando o nível crítico; 72 e 78, alerta, acima do ponto crítico; 78 e 82, perigo; e superior a 82 , emergência.

Amostras de sangue foram coletadas quinzenalmente de dois animais de cada tratamento para execução do hemograma, conforme metodologia descrita por Vallada (1988) e dosagem da concentração de glicose, segundo metodologia de sistema enzimático Glicose GOD-ANA, Labtest.

As análises estatísticas consideraram que os tratamentos foram arranjados em um esquema fatorial $2 \times 2$ (dois regimes alimentares e dois sistemas de pastejo), sendo os dados provenientes de delineamento em parcelas subdivididas, em que os animais representaram as parcelas e os dias de medições, as subparcelas, com o seguinte modelo:

$$
\begin{gathered}
\text { Yijklm }=\mathrm{m}+\mathrm{RAi}+\mathrm{SPj}+(\mathrm{RAxSP}) \mathrm{ij}+ \\
\mathrm{A}\left(\mathrm{RA}^{*} \mathrm{SP}\right) \mathrm{ijk}+\mathrm{Dl}+\left(\mathrm{RA}^{*} \mathrm{D}\right) \mathrm{il}+(\mathrm{SP} * \mathrm{D}) \mathrm{jl}+ \\
\left(\mathrm{RA}^{*} \mathrm{SP} \mathrm{D}^{*}\right) \mathrm{ij} 1+\text { eijklm }
\end{gathered}
$$

em que: Yijklm é o valor relativo ao animal $\mathrm{k}$ que recebeu o regime alimentar i no sistema de pastejo $j$ no tempo 1; $\mathrm{m}$, a média geral; RAi, o efeito do regime alimentar $\mathrm{i} ; \mathrm{SPj}$, o efeito do sistema de pastejo $\mathrm{j}$; (RA*SP)ij, o efeito da interação regime alimentar $i$ com o sistema de pastejo j; A(RA*SP)ijk, o efeito do animal $\mathrm{k}$, do regime alimentar i e sistema de pastejo j; D1, o efeito do dia de medição 1; (RA*D)il, o efeito da interação entre o regime alimentar i e o dia de medição 1; (SP*D)jl, o efeito da interação entre o sistema de produção j e o dia de medição 1 ; (RA*SP*D)ijl, o efeito da interção entre o regime alimentar i, o sistema de pastejo j e p dia de medição 1; eijklm, o erro aleatório associado a cada observação.

A análise de variância foi realizada pelo procedimento GLM (SAS, 2000) e as médias obtidas, comparadas pelo teste Tukey a $5 \%$ de significância,

\section{Resultados e Discussão}

As condições meteorológicas médias, ao longo do período experimental, resultaram em valores mais altos no período da tarde (Tabela 1).

A temperatura do ar (TBS) média pela manhã $\left(23,19^{\circ} \mathrm{C}\right)$ encontrou-se abaixo da temperatura máxima admissível de $32^{\circ} \mathrm{C}$, recomendada para produção mínima de cabras lactantes, descrita por Stevens et al. (1974), citado por Nääs (1986), e da temperatura crítica superior de 30 a $35^{\circ} \mathrm{C}$ descrita por $\mathrm{Lu}(1990)$, enquanto no período da tarde $\left(35,50^{\circ} \mathrm{C}\right)$ encontrou-se superior ao limite estabelecido por estes autores. Levando-se em consideração o índice de temperatura e umidade (ITU), registrou-se tanto pela manhã $(54,31)$ como à tarde $(66,48)$ condição de ausência de estresse (ITU < 70), segundo Hahn (1985), embora os valores máximos de ITU no período da tarde tenham se apresentado elevados, o que contrasta também com valores elevados de temperatura do ar.

\begin{tabular}{|c|c|c|c|}
\hline \multirow[t]{2}{*}{$\begin{array}{l}\text { Variáveis } \\
\text { Variables }\end{array}$} & & \multicolumn{2}{|c|}{$\begin{array}{c}\text { Período } \\
\text { Period }\end{array}$} \\
\hline & & $\begin{array}{l}\text { Manhã } \\
\text { Morning }\end{array}$ & $\begin{array}{c}\text { Tarde } \\
\text { Afternoon }\end{array}$ \\
\hline Temperatura do ar $\left({ }^{\circ} \mathrm{C}\right)(\mathrm{TBS})$ & Média (Average) & 23,19 & 27,65 \\
\hline \multirow[t]{2}{*}{ Air temperature $\left({ }^{\circ} \mathrm{C}\right)(\mathrm{DBT})$} & Mínima (Minimum) & 14,00 & 18,25 \\
\hline & Máxima (Maximum) & 29,75 & 35,50 \\
\hline Temperatura do Globo $\left({ }^{\circ} \mathrm{C}\right)(\mathrm{TG})$ & Média (Average) & 30,25 & 35,31 \\
\hline \multirow[t]{2}{*}{ Globe temperature $\left({ }^{\circ} \mathrm{C}\right)(\mathrm{GT})$} & Mínima (Minimum) & 17,60 & 17,00 \\
\hline & Máxima (Maximum) & 40,55 & 46,43 \\
\hline Velocidade do vento (m/s) (VV) & Média (Average) & 0,88 & 0,90 \\
\hline \multirow[t]{2}{*}{ Air velocity $(\mathrm{m} / \mathrm{s})(A V)$} & Mínima (Minimum) & 0,10 & 0,25 \\
\hline & Máxima (Maximum) & 3,56 & 2,88 \\
\hline Umidade relativa do ar (\%) (UR) & Média (Average) & 82,89 & 73,28 \\
\hline \multirow[t]{2}{*}{ Relative humidity (\%) (RH) } & Mínima (Minimum) & 60,10 & 49,27 \\
\hline & Máxima (Maximum) & 97,02 & 98,63 \\
\hline Índice de temperatura e umidade (ITU) & Média (Average) & 54,31 & 66,48 \\
\hline \multirow[t]{2}{*}{ Temperature-humidity index (THI) } & Mínima (Minimum) & 36,40 & 34,50 \\
\hline & Máxima (Maximum) & 83,30 & 96,80 \\
\hline
\end{tabular}

Tabela 1 - Valores mínimos, médios e máximos das variáveis meteorológicas ao longo do período experimental Table 1 - Minimum, average and maximum values of meteorological variables during the experimental period

R. Bras. Zootec., v.31, n.3, p.1245-1250, 2002 
Os valores médios dos constituintes sangüíneos avaliados durante o período experimental estão apresentados, respectivamente, na Tabela 2 e na Figura 1.

Consta na Tabela 3 a análise de variância dos dados de glicose, hemoglobina e hematócrito, obtidos durante o experimento.

Observa-se que o regime alimentar influenciou $(\mathrm{P}<0,05)$ a concentração de glicose, a concentração de hemoglobina e o hematócrito, enquanto o sistema de produção apresentou efeito $(\mathrm{P}<0,05)$ apenas para a concentração de $\mathrm{Hb}$ e o Ht.

Não foram encontradas diferenças na concentração de glicose e hemoglobina e no hematócrito,

Tabela 2 - Média dos valores observados ao longo do período experimental para concentração de glicose e hemoglobina e da porcentagem de hematócrito

Table 2 - Average of values observed during the experimental period for the glucose concentration, hemoglobin and percentage of hematocrit

\begin{tabular}{lc}
\hline $\begin{array}{l}\text { Variáveis } \\
\text { Variables }\end{array}$ & $\begin{array}{c}\text { Média } \\
\text { Average }\end{array}$ \\
\hline $\begin{array}{l}\text { Glicose }(\mathrm{mg} / 100 \mathrm{~mL}) \\
\text { Glucose }(\mathrm{mg} / 100 \mathrm{~mL})\end{array}$ & 54,74 \\
Hemoglobina $(\mathrm{g} / 100 \mathrm{~mL})$ & 7,00 \\
Hemoglobin $(\mathrm{g} / 100 \mathrm{~mL})$ & \\
Hematócrito $(\%)$ & 20,68 \\
Hematocrit $(\%)$ & \\
\hline
\end{tabular}

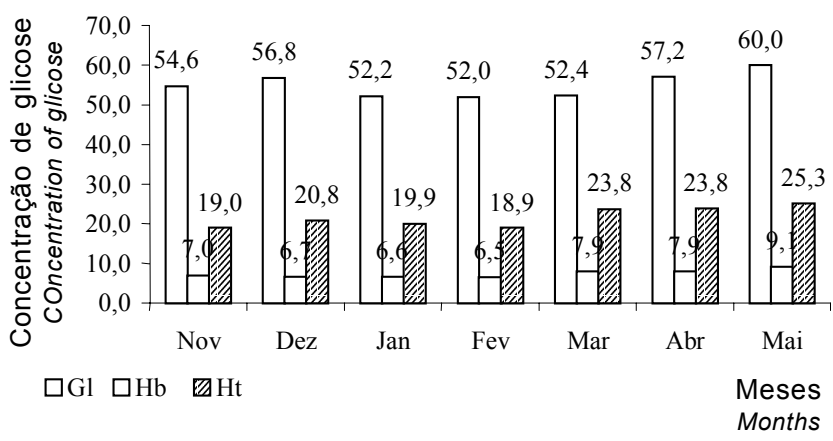

Figura 1 - Comportamento das concentrações de glicose ( $\mathrm{Gl}-\mathrm{g} / 100 \mathrm{~mL})$ e hemoglobina $(\mathrm{Hb}-\mathrm{g} /$ $100 \mathrm{~mL}$ ) e da porcentagem do hematócrito $(\mathrm{Ht}-\%)$, durante o período experimental.

Figure 1 - Concentrations of glucose (Gl - g/100 mL) and hemoglobin $(\mathrm{Hb}-\mathrm{g} / 100 \mathrm{~mL})$ and hematocrit (Ht - \%) during the experimental period. quando os animais foram submetidos aos diferentes regimes (Tabela 4). Embora não tenham sido detectadas diferenças entre estes regimes alimentares, nota-se que os maiores valores para glicose, hemoglobina e hematócrito foram encontrados no regime $\mathrm{R} 2$, causados pela maior quantidade de concentrado consumido $(75,13 \mathrm{~kg})$, que os animais do regime alimentar R1 $(55,82 \mathrm{~kg})$. Assim, é de se esperar que, em função de maior disponibilidade de alimento facilmente degradado a nível de rúmen, o regime alimentar R2 possa ter contribuído para a elevação da taxa de glicose, fornecendo ao seu organismo, pela degradação do amido, no processo digestivo, maior quantidade de glicose. Segundo Andrigueto (1990), quando o organismo recebe quantidades abundantes de glicídios alimentares, ele estoca provisória e parcialmente a glicose, na forma de glicogênio, do qual é devolvida progressivamente à circulação.

Segundo Gütler et al. (1986), os eritrócitos, células especializadas no transporte de $\mathrm{O}_{2}$ e $\mathrm{CO}_{2}$, que contêm como principal componente a hemoglobina, apresentam nas cabras os mais altos valores eritrocitários entre os animais domésticos, pois possuem glóbulos vermelhos muitos pequenos. Estes mesmos autores citaram que a alimentação carente reduz a neoformação de eritrócitos assim como o valor do hematócrito; como a hemoglobina representa aproximadamente $92 \%$ dos componentes orgâni$\cos$ dos eritrócitos, ocorre também redução da hemoglobina. Assim, para a $\mathrm{Hb}$, o regime alimentar R2 aparentemente foi mais deficiente. Isto demonstra que, para animais em lactação e mantidos em pastagem, é necessário haver equilíbrio no fornecimento de alimento, impedindo que os animais façam uso de suas reservas corporais.

Analisando o sistema de produção, as diferenças ocorreram apenas para a concentração de hemoglobina e a porcentagem do hematócrito. Os valores de $\mathrm{Hb}$ e $\mathrm{Ht}$ encontrados nos animais do sistema de produção $\mathrm{S} 1(7,60 \mathrm{~g} / 100 \mathrm{~mL}, 22,35 \%) \mathrm{e}$ do sistema de produção $\mathrm{S} 2(6,05 \mathrm{~g} / 100 \mathrm{~mL}, 17,72 \%)$ (Tabela 4) estão, respectivamente, próximos e abaixo dos valores mínimos $(7 \mathrm{~g} / 100 \mathrm{~mL}$ e $21 \%$ ) para caprinos, segundo Gütler et al. (1986). Segundo esses autores, a proporção de volume das células sangüíneas é dependente da solicitação física dos animais, sendo que animais sob esforço físico maior possuem valor de hematócrito mais elevado que aqueles sob menor esforço físico. Sabendo-se que a

R. Bras. Zootec., v.31, n.3, p.1245-1250, 2002 
Tabela 3 - Resumo da análise de variância dos dados de glicose (GI), hemoglobina $(\mathrm{Hb})$ e hematócrito $(\mathrm{Ht})$, em função do regime alimentar e do sistema de pastejo

Table 3 - Analysis of variance of glucose, (Gl), hemoglobin $(H b)$ and hematocrit $(H t)$ data in function of diet and production system

\begin{tabular}{|c|c|c|c|c|}
\hline \multirow{3}{*}{$\begin{array}{l}\text { Fonte de variação } \\
\text { Source of variation }\end{array}$} & \multicolumn{4}{|c|}{$\begin{array}{l}\text { Quadrado médio } \\
\text { Mean square }\end{array}$} \\
\hline & GL & G & $\mathrm{Hb}$ & $\mathrm{Ht}$ \\
\hline & $D F$ & $G l$ & $H b$ & $H t$ \\
\hline Regime alimentar (RA) & 1 & $59,52 * *$ & $3,63 * *$ & $44,34 * *$ \\
\hline \multicolumn{5}{|l|}{ Diet } \\
\hline Sistema de produção (SP) & 1 & 12,07 & $23,40 * *$ & $, 220,01 * *$ \\
\hline \multicolumn{5}{|l|}{ Production system } \\
\hline $\mathrm{RA} * \mathrm{SP}$ & 1 & 23,14 & $7,72 * *$ & $30,01 * *$ \\
\hline Animal (RA*SP) & 4 & $90,77 * *$ & $1,94 * *$ & $15,25 * *$ \\
\hline \multicolumn{5}{|l|}{ Animal } \\
\hline $\operatorname{Dia}(\mathrm{D})$ & 13 & $33,47 * *$ & $1,41 * *$ & $17,54 * *$ \\
\hline \multicolumn{5}{|l|}{ Day } \\
\hline $\mathrm{RA} * \mathrm{D}$ & 12 & $17,30^{*}$ & 0,68 & $8,11^{* *}$ \\
\hline $\mathrm{SP} * \mathrm{D}$ & 6 & 16,65 & $1,09 *$ & $8,39 *$ \\
\hline $\mathrm{RA} * \mathrm{SP} * \mathrm{D}$ & 6 & 8,64 & 0,13 & 4,39 \\
\hline Resíduo & 7,48 & 0,41 & 2,60 & \\
\hline \multicolumn{5}{|l|}{ Error } \\
\hline GL Resíduo & 33 & & & \\
\hline \multicolumn{5}{|l|}{ DF Error } \\
\hline $\mathrm{CV}(\%)$ & & 5,02 & 9,14 & 7,78 \\
\hline
\end{tabular}

$\mathrm{GI}=$ grau de liberdade, $\mathrm{CV}=$ coeficiente de variação, ${ }^{* *}(\mathrm{P}<0,01),{ }^{*}(\mathrm{P}<0,05)$.

$D F=$ Degree of freedom, $C V=$ Coefficient of variation, ${ }^{* *}(P<.01),{ }^{*}(P<.05)$.

Tabela 4 - Médias estimadas dos valores de glicose $(\mathrm{Gl})$, hemoglobina $(\mathrm{Hb})$ e hematócrito $(\mathrm{Ht})$ Table 4 - Estimate averages of glucose (Gl), hemoglobin $(\mathrm{Hb})$ and hematocrit $(\mathrm{Ht})$ values

\begin{tabular}{|c|c|c|c|}
\hline & $\begin{array}{l}\mathrm{Gl}(\mathrm{mg} / 100 \mathrm{~mL}) \\
G l(\mathrm{mg} / 100 \mathrm{~mL})\end{array}$ & $\begin{array}{l}\mathrm{Hb}(\mathrm{g} / 100 \mathrm{~mL}) \\
\mathrm{Hb}(\mathrm{g} / 100 \mathrm{~mL})\end{array}$ & $\begin{array}{l}\mathrm{Ht}(\%) \\
\mathrm{Ht}(\%)\end{array}$ \\
\hline $\begin{array}{l}\text { Sistema do produçã } \\
\text { Production system }\end{array}$ & & & \\
\hline $\begin{array}{l}\text { Pastagem } \\
\text { Pasture }\end{array}$ & $54,24^{\mathrm{a}}$ & $7,60^{\mathrm{a}}$ & $22,42^{\mathrm{a}}$ \\
\hline $\begin{array}{l}\text { Semiconfinamento } \\
\text { Semiconfined } \\
\text { Regime alimentar } \\
\text { Diet }\end{array}$ & $54,67^{\mathrm{a}}$ & $6,05^{b}$ & $17,71^{b}$ \\
\hline $\begin{array}{l}30: 30 \\
30: 30\end{array}$ & $53,50^{\mathrm{a}}$ & $6,68^{a}$ & $19,65^{\mathrm{a}}$ \\
\hline $\begin{array}{l}60: 15 \\
60: 15\end{array}$ & $55,34^{\mathrm{a}}$ & $7,42^{\mathrm{a}}$ & $21,86^{\mathrm{a}}$ \\
\hline
\end{tabular}

Médias, nas colunas, seguidas de letras diferentes diferem pelo teste de Tukey a $5 \%$. Averages, in the column, followed by different letters, are different by Tukey test a $5 \%$.

função da hemoglobina consiste no transporte de oxigênio dos pulmões para os diferentes tecidos e que durante o esforço físico a liberação de oxigênio via hemoglobina é mais rápida, em função da elevação na taxa de consumo de oxigênio (Schimidt-Nielsen, 1996), eram esperados valores maiores de $\mathrm{Hb}$ e $\mathrm{Ht}$ nos animais em pastagem, visto que a freqüência respiratória destes animais foi mais elevada ( $82,00 \mathrm{resp} . / \mathrm{min}$.) que a dos animais semiconfinados (75,22 resp./min.).

Igbokwe et al. (1998), estudando 210 cabras Sahel Nigerianas, encontraram valores de Ht entre 16 e $45 \%$, sendo a maior freqüência entre 26 e $30 \%$, enquanto o teor de $\mathrm{Hb}$ encontrado, entre 3,8 e $11,6 \mathrm{~g} / 100 \mathrm{~mL}$ e o teor mais freqüente, entre 7,1 e $8,0 \mathrm{~g} / 100 \mathrm{~mL}$. 


\section{Conclusões}

O regime alimentar não exerceu efeito nos constituintes sangüíneos, porém maior consumo de concentrado acarretou maiores valores de glicose, hemoglobina e hematócrito.

Animais em pastagem, devido à maior solicitação física, em função da freqüência respiratória elevada, apresentaram maiores valores de hemoglobina e hematócrito em relação a animais semiconfinados.

\section{Literatura Citada}

ANDRIGUETO, J.M. Nutrição animal. São Paulo: Nobel. 1990. 396p.

BACCARI, P.; BRASIL, L.H.A.; TEODORO, S.M. et al. Thermoregulatory responses of alpine goats during thermal stress. In: INTERNATIONAL LIVESTOCK ENVIRONMENT SYMPOSIUM, 5., 1997, Minneapolis. Anais... Minneapolis: 1997. p. 29-31.

GÜNTLER, H.; KETZ, A.; KOLB, E. et al. Fisiologia veterinária. 2.ed. São Paulo: Guanabara. 1986. 569p.

HAHN, G.L. Management and housing of farm animals en hot environments. In: YOUSEF, M.K. (Ed.) Stress physiology in livestock. Boca Raton: CRC Press, 1985. v.2. p.151-174.

IGBOKWE, I.O., RIBADU, A.Y. BUKAR, M.M. Erythrocyte gluthione concentrations in Nigerian Sahel goats. Small Ruminant Research, v.30, p.1-6, 1998.

LEHNINGER, A.L. Princípios da bioquímica. São Paulo: Sarvier, 1984. 725p.
LU, C. Heat stress and goat production. In: SIMPÓSIO INTERNACIONAL DE BIOCLIMATOLOGIA ANIMAL NOS TRÓPICOS: PEQUENOS E GRANDES RUMINANTES, 1., 1986, Fortaleza. Anais... Brasília: EMBRAPA/Departamento de Informação e Editoração, 1990. p.95-105.

NÄÄS, I.A. Princípios de conforto térmico na produção animal. São Paulo: Editora Ícone, 1989. 183p.

NATIONAL RESEARCH COUNCIL - NRC. Nutrients Requirements of goats. Angora, dairy and meat goats in temperate and tropical countries. Washington, D.C.: 1981.91p.

REYNOLDS, C.K.; HARMON, D.L.; CECAVA, M.J. Absorption and delivery of nutrients for milk protein synthesis by portal-drained viscera. Journal of Dairy Science, v.27, p.251-256, 1994.

SAS INSTITUTE. Statistic guide for personal computers. Cary: North Carolina, 2000.

SCHMIDT-NIELSEN, K. Fisiologia animal-adaptação e meio ambiente. 5.ed. São Paulo: Santos, 1996. 546p

SILVA, R.G. Equações para estimativa da carga térmica radiante através do globo de Vernon. In: WORKSHOP DE BIOCLIMATOLOGIA ANIMAL, 2., 1989, Jaboticabal. Anais... Jaboticabal: Universidade Estadual Paulista, 1989. p.1-9.

THOM, E.C. Cooling degree"day air conditioning, heating, and ventilating. Transactions of the American Society Heating Refrigeration Air-Conditionning Engrs, v.55, p.65-72, 1958.

VALLADA, E.P. Manual de técnicas hematológicas. Rio de Janeiro: Athenen, 1988. 423p.

Recebido em: 12/04/01

Aceito em: 18/05/02 